\title{
The effect of diffusioosmosis on water transport in polymer electrolyte fuel cells
}

\author{
Huan J. Keh, Hsien Chen Ma* \\ Department of Chemical Engineering, National Taiwan University, Taipei 10617, Taiwan, ROC
}

\section{A R T I C L E I N F O}

\section{Article history:}

Received 8 January 2008

Received in revised form 27 February 2008

Accepted 27 February 2008

Available online 2 March 2008

\section{Keywords:}

Diffusioosmosis

Electroosmosis

Water transport

Polymer electrolyte membrane

Fuel cell

\begin{abstract}
A B S T R A C T
An analytical study of the effect of diffusioosmosis caused by the concentration gradient of hydrogen ions on the isothermal transport of water in a fully hydrated membrane of a polymer electrolyte fuel cell (PEFC) is presented. A capillary tube or slit with a negatively charged wall is chosen to model the nanopores of the membrane. The electric double layer adjacent to the capillary wall may have an arbitrary thickness relative to the capillary radius and its electrostatic potential distribution is determined as the solution of the Poisson-Boltzmann equation. Solving a modified Navier-Stokes equation, the fluid velocity in the axial direction of the capillary induced by the macroscopic electric field and protonic concentration gradient is obtained as a function of the radial position in closed forms. The results for the local and averaged electrokinetic velocities in the capillary show that the effect of diffusioosmosis on the water transport in the membrane of a PEFC can be significant in comparison with that of electroosmosis under low-potential-difference operations.
\end{abstract}

(c) 2008 Elsevier B.V. All rights reserved.

\section{Introduction}

The polymer electrolyte fuel cell (PEFC) uses a polymeric membrane as an electrolyte, analogous in acidity to the electrolyte in the automotive battery, but dimensionally fixed. This facilitates sealing in the production process and furnishes both cell and stack with longevity. PEFCs can generate high electric-current density and allow quick start-ups and instant response to changes in the demand of power. Being efficient, environmentally clean, and potentially low-cost power systems for both stationary and mobile applications, PEFCs are of interest to both electric vehicle and portable electronics manufacturers [1-3].

The polymer electrolyte membrane (PEM), which prevents mixing of feed gases and provides transport of protons from the anode to the cathode, is one of the key components in PEFC systems. Perfluorosulfonic acid (PFSA) copolymer membranes, such as DuPont's Nafion (composed of carbon-fluorine backbone chains with perfluoro side chains terminated by negatively charged sulfonic groups), are the most commonly used PEMs in PEFCs operating at low temperatures below $80^{\circ} \mathrm{C}$. Some high-temperature PEMs for operation above $100^{\circ} \mathrm{C}$, such as naphthalene-based sulfonated polyimide (SPI) and modified PFSA membranes, are also under active development $[4,5]$.

\footnotetext{
* Corresponding author. Tel.: +8862 33663015; fax: +8862 23623040

E-mail address: hcma01@ntu.edu.tw (H.C. Ma).
}

The performance of a PEFC is strongly influenced by the protonic conductivity of its PEM, and the conductivity is highly dependent on the state of hydration of the membrane. The sources of water in the membrane are the humidified reactant gases and the product of the chemical reaction of hydrogen ions and oxygen occurred at the cathode. Undoubtedly, the protonic conductivity of a PEM increases with its content of water. But, an excess of water in the fuel cell may lead to electrode flooding and thus the diffusion of the reactant gases will be suppressed. In addition, external humidification of the feed gases is a burden for the fuel cell. Therefore, understanding of the water transport and successful management of the water in the PEM is critical to optimize the performance of PEFCs [6-8].

The water distribution throughout a PEFC is determined by the interplay among the water uptake by the PEM, the rate of chemical reactions at the electrodes, the diffusion of water due to its activity gradients, and the water transport in the membrane accompanied by the protonic current from the anode to the cathode. The protonic current produces water flow along the charged nanopore walls in the membrane by two electrokinetic mechanisms. The first is electroosmosis resulting from the interaction between the macroscopic electric field and the electric double layer adjacent to the pore wall, and the effects on the water transport caused by this well-known mechanism were studied extensively in the past [9-14]. The second mechanism is diffusioosmosis (also known as capillary osmosis) comprising the stresses developed by the tangential gradient of the excess pressure within the double layer [15-21], and this effect on the water transport in the PEM has not been investigated yet. 


\begin{tabular}{|c|c|}
\hline \multicolumn{2}{|c|}{ Nomenclature } \\
\hline$e$ & elementary charge $(\mathrm{C})$ \\
\hline E & macroscopic electric field $\left(\mathrm{V} \mathrm{m}^{-1}\right)$ \\
\hline $\bar{E}$ & $=E e / \kappa k T$ \\
\hline$h$ & half thickness of a slit (m) \\
\hline$k$ & Boltzmann's constant $\left(\mathrm{J} \mathrm{K}^{-1}\right)$ \\
\hline$L$ & length of a capillary (m) \\
\hline$n$ & concentration distribution of hydrogen ions $\left(\mathrm{m}^{-3}\right)$ \\
\hline$n^{\infty}$ & $\begin{array}{l}\text { bulk concentration distribution of hydrogen ions } \\
\left(\mathrm{m}^{-3}\right)\end{array}$ \\
\hline $\bar{N}$ & $=\left|\nabla n^{\infty}\right| / \kappa n^{\infty}(0)$ \\
\hline$p$ & pressure distribution $\left(\mathrm{N} \mathrm{m}^{-2}\right)$ \\
\hline$r$ & radial coordinate in a tube $(\mathrm{m})$ \\
\hline$R$ & radius of a tube $(\mathrm{m})$ \\
\hline$T$ & absolute temperature $(\mathrm{K})$ \\
\hline$u$ & fluid velocity distribution $\left(\mathrm{m} \mathrm{s}^{-1}\right)$ \\
\hline$\langle u\rangle$ & average fluid velocity $\left(\mathrm{m} \mathrm{s}^{-1}\right)$ \\
\hline$U^{*}$ & characteristic electrokinetic velocity $\left(\mathrm{m} \mathrm{s}^{-1}\right)$ \\
\hline$y$ & lateral coordinate in a slit $(\mathrm{m})$ \\
\hline$z$ & axial coordinate in a capillary (m) \\
\hline \multicolumn{2}{|c|}{ Greek letters } \\
\hline$\gamma$ & $=\ln (\kappa r)$ \\
\hline$\varepsilon$ & dielectric permittivity $\left(\mathrm{C}^{2} \mathrm{~J}^{-1} \mathrm{~m}^{-1}\right)$ \\
\hline$\underline{\zeta}$ & zeta potential of capillary walls (V) \\
\hline$\frac{2}{\zeta}$ & $=\zeta e / k T$ \\
\hline$\eta$ & fluid viscosity $\left(\mathrm{kg} \mathrm{m}^{-1} \mathrm{~s}^{-1}\right)$ \\
\hline$\kappa$ & Debye screening parameter $\left(\mathrm{m}^{-1}\right)$ \\
\hline$v$ & $=-\bar{\psi}+2 \ln (\kappa r)$ \\
\hline$\sigma$ & surface charge density $\left(\mathrm{C} \mathrm{m}^{-2}\right)$ \\
\hline$\psi$ & electrostatic potential distribution (V) \\
\hline $\bar{\psi}$ & $=\psi e / k T$ \\
\hline $\bar{\psi}_{0}$ & $\bar{\psi}$ at the axis of a capillary \\
\hline
\end{tabular}

The fluid flow resulting from the interaction between a solid wall and a tangential solute concentration gradient is termed diffusioosmosis. The ionic solute-charged wall interaction in diffusioosmosis is electrostatic in nature and its range is the Debye screening length $\kappa^{-1}$ (defined by Eq. (4)). The fluid motion caused by diffusioosmosis has been analytically examined for ionic solutions near a plane wall $[22,23]$ and inside a capillary pore [23-27]. Some experimental results and interesting applications concerning diffusioosmosis are also available in the literature [28]. Ionic solutions with a concentration gradient of order $100 \mathrm{kmol} \mathrm{m}^{-4}\left(=1 \mathrm{M} \mathrm{cm}^{-1}\right)$ along solid surfaces with a zeta potential of order $k T / e(\sim 25 \mathrm{mV}$; $e$ is the charge of a proton, $k$ is the Boltzmann constant, and $T$ is the absolute temperature) can flow by diffusioosmosis at a velocity of several micrometers per second.

The pores in the membrane (e.g., Nafion) of a PEFC can be simulated as straight nanochannels with negatively charged walls, and the hydrogen ions generated at the anode create a protonic concentration gradient in the membrane against the cathode; as a result, diffusioosmosis will contribute to the flow of water. In

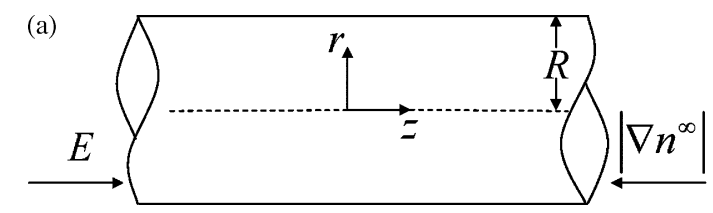

this work, we will analytically study the effect of diffusioosmosis, in comparison with that of electroosmosis, on the isothermal transport of water in a fully hydrated membrane whose shapes of channels are maintained during the operation of the fuel cell. The capillary channels are in general of the order nanometer in radius, and no assumption is made about the thickness of the electric double layers adjacent to the capillary walls or the magnitude of the zeta potential of the walls.

\section{Electrokinetic flow in a capillary tube}

In this section, we consider the combined electroosmotic and diffusioosmotic flow of a fluid solution of hydrogen ions in a straight capillary tube of radius $R$ and length $L$ with $R \ll L$ in the presence of an external electric field with a constant magnitude $E$ in the axial $(z)$ direction, as illustrated in Fig. 1a, at the steady state. The discrete nature of the surface charges, which are negative and uniformly distributed over the capillary wall, is ignored. The prescribed concentration gradient $\nabla n^{\infty}$ of the hydrogen ions has a constant magnitude (equal to $\left|\nabla n^{\infty}\right|$ ) along the $-z$ direction, where $n^{\infty}(z)$ is the linear concentration distribution of the hydrogen ions in the bulk solution phase in equilibrium with the fluid inside the capillary. The hydrogen ions can diffuse in the capillary, so there exists no regular osmotic flow of the solvent. It is assumed that $L\left|\nabla n^{\infty}\right| / n^{\infty}(0)<<1$, where $z=0$ is set at the midpoint through the capillary axially. Thus, the variation of the electrostatic potential and ionic concentration in the electric double layer (without co-ions) adjacent to the capillary wall with the axial position can be neglected.

\subsection{Electrostatic potential distribution}

We first deal with the electrostatic potential distribution in the ionic solution on a cross-section of the capillary tube. If $\psi(r)$ represents the electrostatic potential at a point with distance $r$ from the axis of the tube (where the total electric potential equals $\psi$-Ez from the linear superposition of $\psi$ that would exist in the absence of the imposed electric field and $-E z$ that arises from this electric field) relative to that in a neutral bulk solution and $n(r, z)$ denotes the local concentration of the hydrogen ions, then Poisson's equation gives

$\frac{1}{r} \frac{\mathrm{d}}{\mathrm{d} r}\left(r \frac{\mathrm{d} \psi}{\mathrm{d} r}\right)=-\frac{e}{\varepsilon} n(r, 0)$.

Here $\varepsilon$ is the dielectric permittivity of the ionic solution, which is taken to be a constant. The local concentration of hydrogen ions can also be related to the electrostatic potential by the Boltzmann equation:

$n=n^{\infty} \mathrm{e}^{-\bar{\psi}}$

where $\bar{\psi}=\psi e / k T$ is the normalized electrostatic potential distribution.

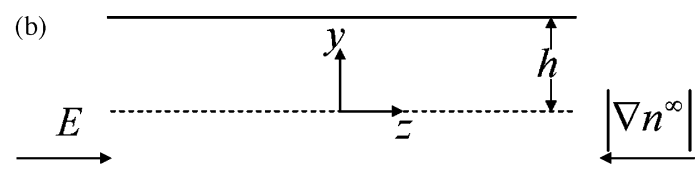

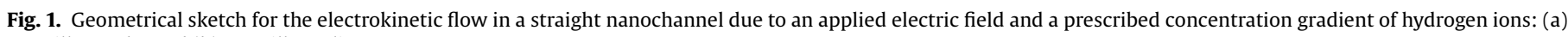
a capillary tube and (b) a capillary slit. 
Substitution of Eq. (2) into Eq. (1) leads to the Poisson-Boltzmann equation:

$\frac{1}{r} \frac{\mathrm{d}}{\mathrm{d} r}\left(r \frac{\mathrm{d} \bar{\psi}}{\mathrm{d} r}\right)=-\kappa^{2} \mathrm{e}^{-\bar{\psi}}$,

where $\kappa$ is the reciprocal of the Debye screening length defined by

$\kappa=\left[\frac{n^{\infty}(0)}{\varepsilon k T}\right]^{1 / 2} e$.

The boundary conditions for $\bar{\psi}$ are

$r=0: \quad \frac{\mathrm{d} \bar{\psi}}{\mathrm{d} r}=0$,

$r=R: \quad \bar{\psi}=\bar{\zeta}$

where the constant $\bar{\zeta}=\zeta e / k T$ is the dimensionless zeta potential at the shear plane of the capillary wall adjacent to the fluid solution having a uniform bulk concentration $n^{\infty}(0)$ of the hydrogen ions.

Introducing the variables $\gamma=\ln (\kappa r)$ and $v=-\bar{\psi}+2 \ln (\kappa r)$, we can rewrite Eq. (3) as

$\frac{\mathrm{d}}{\mathrm{d} \gamma}\left(\frac{\mathrm{d} v}{\mathrm{~d} \gamma}\right)^{2}=2 \frac{\mathrm{de}^{v}}{\mathrm{~d} \gamma}$.

The integration of the above equation with respect to $\gamma$ subject to boundary condition ( $5 \mathrm{a}$ ) leads to

$\left(\frac{\mathrm{d} v}{\mathrm{~d} \gamma}\right)^{2}=2 \mathrm{e}^{v}+4$

After eliminating the unreasonable root, Eq. (7) becomes

$\frac{\mathrm{d} v}{\mathrm{~d} \gamma}=\left(2 \mathrm{e}^{\nu}+4\right)^{1 / 2}$

By integrating the above equation with respect to $\gamma$ again subject to boundary condition ( $5 \mathrm{~b}$ ), the electrostatic potential distribution can be obtained as

$\bar{\psi}=2 \ln (\kappa r)-\ln \left[-2 \operatorname{sech}^{2}\left(\ln \frac{\kappa r}{2 \sqrt{-2}}-\frac{\bar{\psi}_{0}}{2}\right)\right]$,

where

$\bar{\psi}_{0}=\bar{\psi}(0)=2 \ln \frac{\kappa R}{2 \sqrt{-2}}+\ln \frac{2+\sqrt{4+2 \mathrm{e}^{-\bar{\zeta}}(\kappa R)^{2}}}{2-\sqrt{4+2 \mathrm{e}^{-\bar{\zeta}(\kappa R)^{2}}}}$,

representing the dimensionless electrostatic potential on the axis of the capillary tube.

If the constant surface charge density $\sigma$, instead of the surface potential $\zeta$, is known at the capillary wall, the boundary condition specified by Eq. (5b) should be replaced by the Gauss condition:

$r=R: \quad \frac{\mathrm{d} \bar{\psi}}{\mathrm{d} r}=\frac{e \sigma}{\varepsilon k T}$

The solution for $\bar{\psi}$ given by Eqs. (9a) and (9b) still holds for this condition, with the relation between $\bar{\zeta}$ and $\sigma$ for an arbitrary value of $\kappa R$ as

$\bar{\zeta}=\ln \frac{2(\kappa R)^{2}}{((e \sigma R / \varepsilon k T)-2)^{2}-4}$.

For the hydrogen ionic solution in a capillary tube with a given radius, the above equation predicts that the magnitude of $\zeta$ decreases with an increase in $\kappa R$ for the case of constant surface charge density and the magnitude of $\sigma$ increases with an increase in $\kappa R$ for the case of constant surface potential.

Fig. 2 shows the result of the dimensionless electrostatic potential $\bar{\psi}$ calculated from Eq. (9a) and (9b) as functions of the relative position $r / R$ for various values of the dimensionless parameters $\bar{\zeta}$

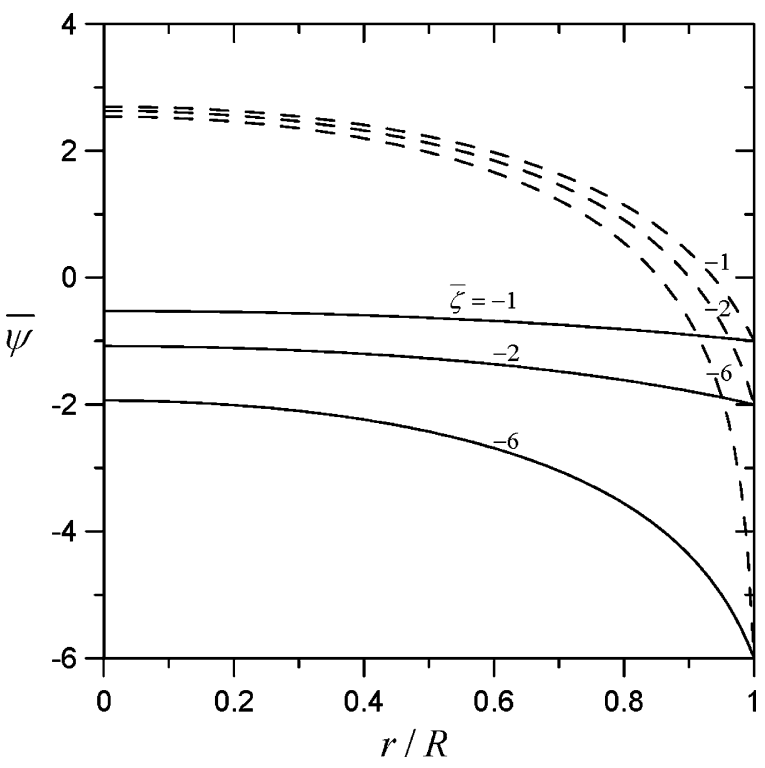

Fig. 2. Plots of the dimensionless potential $\bar{\psi}(r)$ in a capillary tube versus the relative position $r / R$ for various values of $\bar{\zeta}$. The solid and dashed curves denote the cases $\kappa R=1$ and $\kappa R=10$, respectively.

and $\kappa R$. Negative values of $\bar{\zeta}$ are chosen since the capillary wall bears negative charges (e.g., resulting from sulfonic acid $\left(\mathrm{SO}_{3}{ }^{-}\right)$groups attached to the perfluoro side chains of a Nafion carbon-fluorine polymer backbone). As expected, the value of $\bar{\psi}$ is always negative with its value increasing or its magnitude decreasing with a decrease in $r / R$ when the electric double layer adjacent to the capillary wall is relatively thick (say, with $\kappa R<1$ ) for a constant value of $\bar{\zeta}$ at the wall. On the other hand, when the double layer is relatively thin (say, with $\kappa R>2$ ), the value of $\bar{\psi}$ increases with a decrease in $r / R$ from the given dimensionless zeta potential $\bar{\zeta}$ at the wall (with $r / R=1$ ) and becomes positive after $r / R$ is smaller than certain values [29], since the fluid around the axis of the capillary tube still contains hydrogen ions only and the negatively charged wall has little influence on the fluid in this region. For a specified value of $\kappa R$, the value of $\bar{\psi}$ at any position $r / R$ decreases with a decrease in the value of $\bar{\zeta}$. It can be seen that the value of $\bar{\psi}-\bar{\zeta}$ is always positive and increases with an increase in the magnitude of $\bar{\zeta}$ for given values of $r / R$ and $\kappa R$. The increase of $\bar{\psi}-\bar{\zeta}$ with decreasing $r / R$ (which is relatively sharp near the capillary wall) becomes gentler when either $\kappa R$ or the magnitude of $\bar{\zeta}$ is smaller.

\subsection{Fluid velocity distribution}

We now consider the steady electrokinetic flow of the hydrogen ionic solution in the capillary tube. The momentum balances on the Newtonian fluid in the $r$ and $z$ directions give

$\frac{\partial p}{\partial r}+e n \frac{\mathrm{d} \psi}{\mathrm{d} r}=0$

$\frac{\eta}{r} \frac{d}{\mathrm{~d} r}\left(r \frac{\mathrm{d} u}{\mathrm{~d} r}\right)=\frac{\partial p}{\partial z}-e n E$,

where $u(r)$ is the fluid velocity in the positive $z$ direction (axial direction of decreasing ionic concentration), $p(r, z)$ is the pressure distribution, $\eta$ is the viscosity of the fluid, and $E$ is the imposed electric field in the $z$ direction. The boundary conditions for $u$ are

$\begin{array}{ll}r=0: & \frac{\mathrm{d} u}{\mathrm{~d} r}=0, \\ r=R: & u=0 .\end{array}$ 


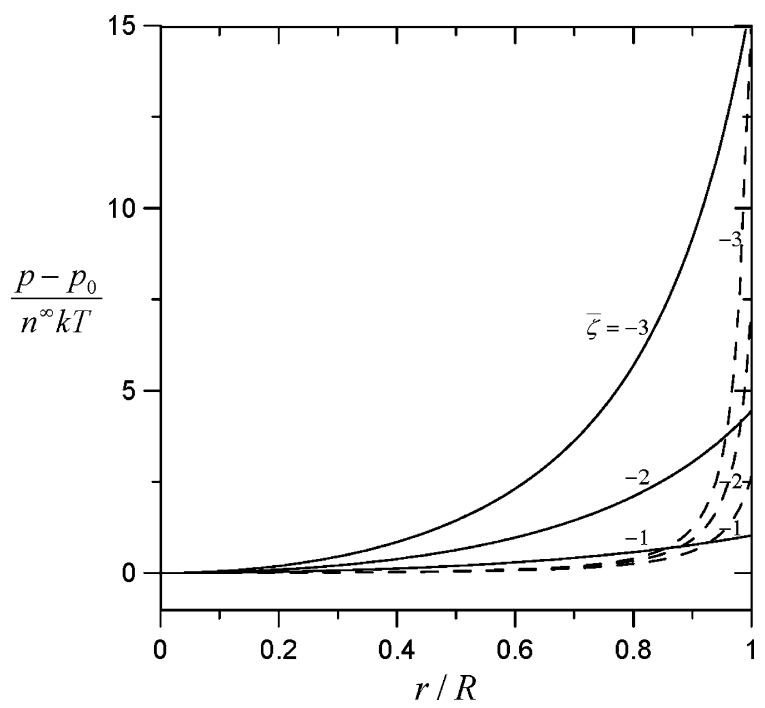

Fig. 3. Plots of the dimensionless pressure $\left(p-p_{0}\right) / n^{\infty} k T$ in a capillary tube versus the relative position $r / R$ for various values of $\bar{\zeta}$. The solid and dashed curves denote the cases $\kappa R=1$ and $\kappa R=10$, respectively.

After the substitution of Eq. (2) into Eq. (12a), the pressure distribution can be determined as

$p=p_{0}+n^{\infty}(z) k T\left[\mathrm{e}^{-\bar{\psi}}-\mathrm{e}^{-\bar{\psi}_{0}}\right]$,

where $p_{0}$ is the pressure on the axis of the capillary tube, which is a constant in the absence of applied pressure gradients and the electrostatic potential distribution $\bar{\psi}(r)$ is given by Eqs. (9a) and (9b). Note that the pressure distribution in the $r$ and $z$ directions results from the variation of the electrostatic potential $\psi$ with $r$ and the variation of the bulk concentration $n^{\infty}$ with $z$, respectively.

Fig. 3 illustrates the result of the normalized pressure $\left(p-p_{0}\right) / n^{\infty} k T$ obtained from Eqs. (14), (9a) and (9b) as functions of the relative coordinate $r / R$ for several values of the dimensionless parameters $\bar{\zeta}$ and $\kappa R$. It can be seen that this normalized pressure is always positive and increases with an increase in $r / R$ irrespective of the values of $\bar{\zeta}$ and $\kappa R$. This behavior is understood by an examination of Eq. (12a) for the fluid momentum balance in the radial direction and knowing that the value of the electrostatic potential $\bar{\psi}$ (which is negative at the capillary wall) decreases with an increase in $r / R$. Accordingly, the value of $\left(p-p_{0}\right) / n^{\infty} k T$ increases with an increase in the magnitude of $\bar{\zeta}$ for given values of $r / R$ and $\kappa R$, and its increase with $r / R$ (which is relatively sharp near the capillary wall) becomes gentler when either $\kappa R$ or the magnitude of $\bar{\zeta}$ is smaller. Eq. (14) also predicts that the axial gradient of the excess pressure in the electric double layer is in the same direction as that of the ionic concentration gradient.

Substituting the ionic concentration distribution of Eq. (2) and the pressure profile of Eq. (14) into Eq. (12b), and then performing the integration with respect to $r$ twice subject to the boundary conditions in Eqs. (13a) and (13b), we obtain

$\frac{u}{U^{*}}=(\kappa R)^{2} \int_{1}^{r / R} \frac{R}{r} \int_{0}^{r / R} \frac{r}{R}\left[\left(\mathrm{e}^{-\bar{\psi}_{0}}-\mathrm{e}^{-\bar{\psi}}\right) \bar{N}-\mathrm{e}^{-\bar{\psi}} \bar{E}\right] d \frac{r}{R} d \frac{r}{R}$,

where the electrostatic potential distribution $\bar{\psi}$ is given by Eqs. (9a) and (9b),

$U^{*}=\frac{n^{\infty}(0) k T}{\eta \kappa}$,

which is a characteristic velocity of the electrokinetic flow of the fluid, $\bar{E}=E e / \kappa k T$ represents a dimensionless strength of the applied electric field to produce the electroosmosis, and
$\bar{N}=\left|\nabla n^{\infty}\right| / \kappa n^{\infty}(0)$ denotes a dimensionless strength of the ionic concentration gradient to cause diffusioosmosis (more accurately, chemiosmosis only). For some typical values, $E \approx 250 \mathrm{~V} \mathrm{~m}^{-1}$, $n^{\infty} /\left|\nabla n^{\infty}\right| \approx 10^{-4} \mathrm{~m}$, and $\kappa^{-1} \approx 10^{-8} \mathrm{~m}$, then both $\bar{E}$ and $\bar{N}$ have the order $10^{-4}$. Eq. (15) indicates that the total fluid velocity is a superposition of the electroosmotic and diffusioosmotic velocities and, under the condition of $\bar{N}=\bar{E}$, the effect of diffusioosmosis is weaker than that of electroosmosis by a factor $1-\mathrm{e}^{\bar{\psi}-\bar{\psi}_{0}}$.

The average fluid velocity $\langle u\rangle$ over a cross-section of the capillary tube is defined as

$\langle u\rangle=2 \int_{0}^{1} u\left(\frac{r}{R}\right) \frac{r}{R} d \frac{r}{R}$,

which can be obtained by numerical integration after the substitution of Eq. (15).

\subsection{Results of fluid velocity and discussion}

The electroosmotic and diffusioosmotic velocities of the hydrogen ionic solution in the capillary tube are given by Eq. (15), in which the dual integration can be performed numerically, setting $\bar{N}=0$ and $\bar{E}=0$, respectively. These velocities in nondimensional mobility form are plotted versus the normalized coordinate $r / R$ in Figs. 4 and 5 for several values of the parameters $\bar{\zeta}$ and $\kappa R$. It can be seen that both the dimensionless electroosmotic mobility $u / U^{*} \bar{E}$ and diffusioosmotic mobility $u / U^{*} \bar{N}$ decrease monotonically with an increase in $r / R$ from maxima at the axis of the capillary $(r / R=0)$ to zero at the capillary wall $(r / R=1)$ due to the no-slip condition at the wall. The fluid flows in the direction of the electric field or decreasing ionic concentration ( $u$ is positive). Both the electroosmotic and diffusioosmotic mobilities are monotonically increasing functions of $\kappa R$ and the magnitude of $\bar{\zeta}$. It implies that a modification of the membrane material in a fuel cell with a smaller pore radius or a lower magnitude of zeta potential could result in less water transport and easier maintenance of the fully hydrated environment. Since the aging of the membrane and the deformation of its nanopores during fuel cell operations often change the values of $\kappa R$ and $\bar{\zeta}$, these factors could affect the consistency of the water transport as well.

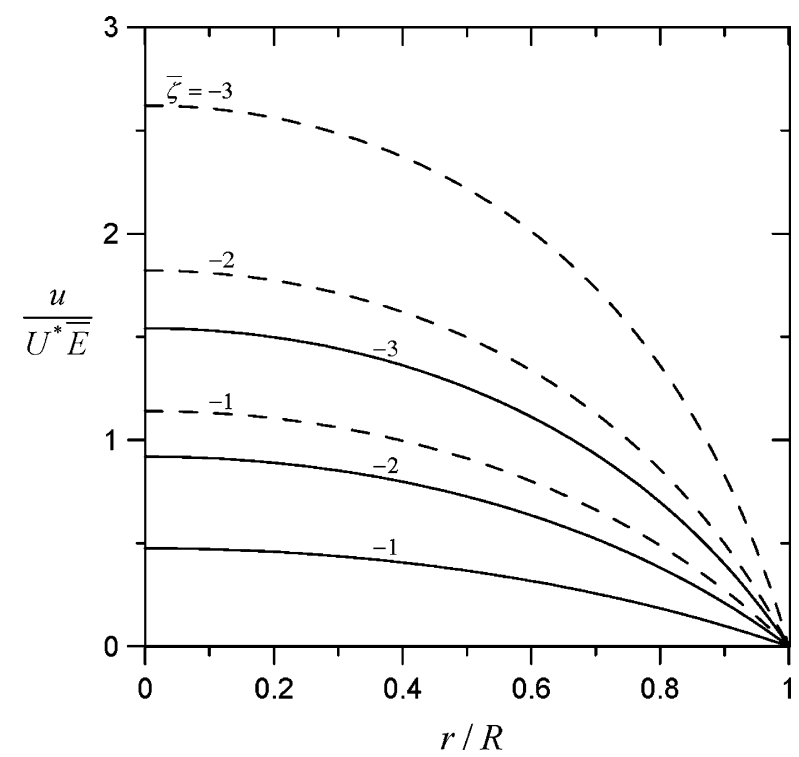

Fig. 4. Plots of the dimensionless electroosmotic mobility $u / U^{*} \bar{E}$ in a capillary tube as calculated from Eq. (15) with $\bar{N}=0$ versus the relative position $r / R$ for various values of $\bar{\zeta}$. The solid and dashed curves denote the cases $\kappa R=1$ and $\kappa R=2$, respectively. 


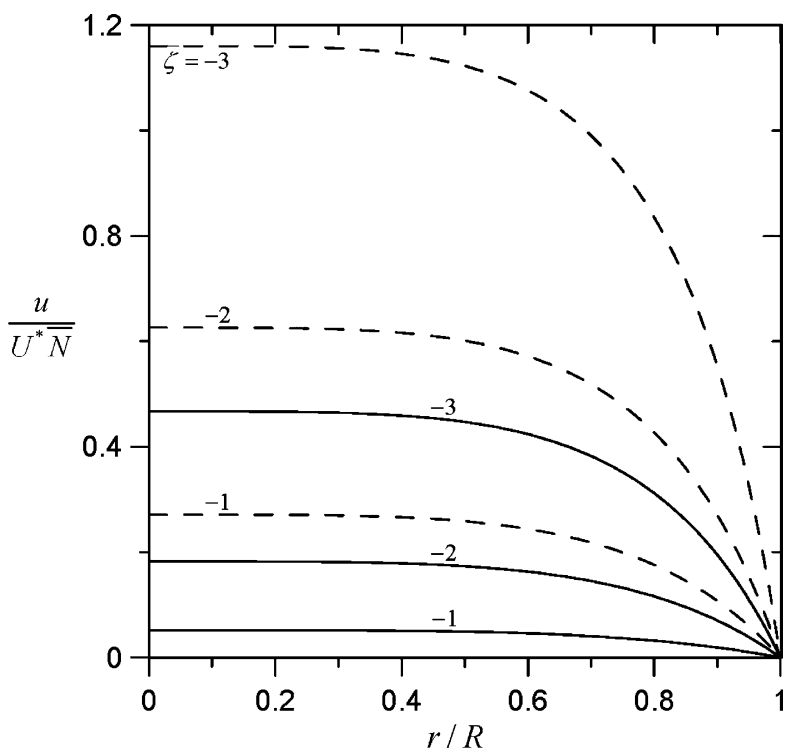

Fig. 5. Plots of the dimensionless diffusioosmotic mobility $u / U^{*} \bar{E}$ in a capillary tube as calculated from Eq. (15) with $\bar{E}=0$ versus the relative position $r / R$ for various values of $\bar{\zeta}$. The solid and dashed curves denote the cases $\kappa R=1$ and $\kappa R=2$, respectively.

In Fig. 6, the dimensionless average electrokinetic velocity $\langle u\rangle / U^{*} \bar{E}$ of the ionic solution in the capillary tube calculated using Eq. (17) is plotted as functions of the parameter $\kappa R$ for some values of the parameters $\bar{N} / \bar{E}$ and $\bar{\zeta}$. The contribution from diffusioosmosis to the total fluid flow increases with the ratio $\bar{N} / \bar{E}$, as expected, and is quite significant (more than 20\%) as this ratio is greater than $1 / 2$. In accordance to the electroosmotic and diffusioosmotic velocity profiles illustrated in Figs. 4 and 5, the average fluid velocity increases with an increase in $\kappa R$ and the magnitude of $\bar{\zeta}$ at any value of $\bar{N} / \bar{E}$.

\section{Electrokinetic flow in a capillary slit}

In this section, we examine the steady electroosmosis and diffusioosmosis of a hydrogen ionic solution in a nanochannel between

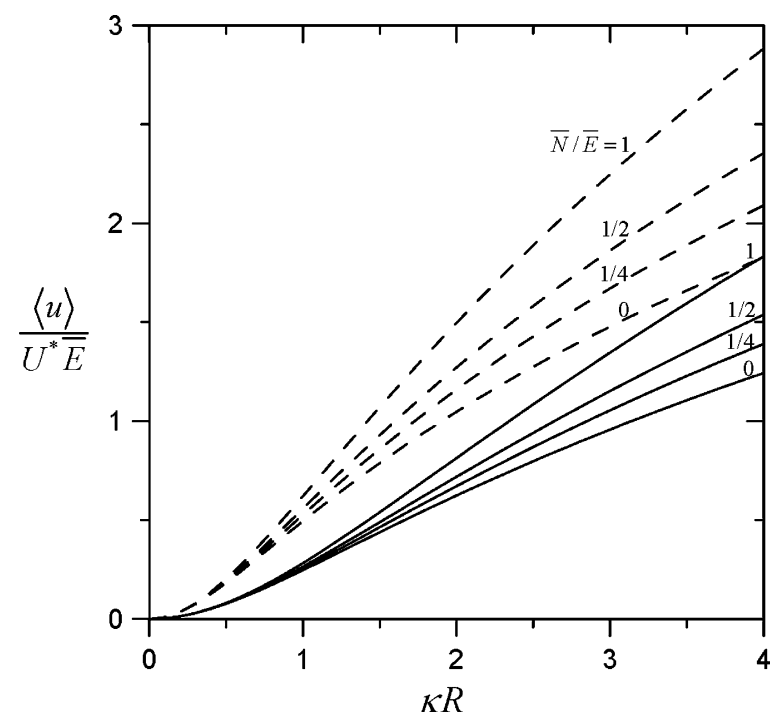

Fig. 6. Plots of the dimensionless average electrokinetic velocity $\langle u\rangle / U^{*} \bar{E}$ in a capillary tube as calculated from Eq. (17) versus the electrokinetic radius $\kappa R$ for various values of $\bar{N} / \bar{E}$. The solid and dashed curves denote the cases $\bar{\zeta}=-1$ and $\bar{\zeta}=-2$, respectively. two identical parallel plates with a separation distance $2 h$ in the presence of a uniform external electric field $E$ in the tangential $z$ direction, as shown in Fig. 1b. The imposed concentration gradient $\nabla n^{\infty}$ of the hydrogen ions is along the $-z$ direction. Owing to the planar symmetry of the system, we need consider only the half region $0 \leq y \leq h$, where $y$ is the distance from the median plane between the slit walls in the normal direction. The analysis for this case is similar to that for the case of electrokinetic flow in a capillary tube presented in the previous section.

\subsection{Electric potential distribution}

For the ionic solution in the capillary slit, let $\psi(y)$ be the electrostatic potential at the position $y$ and $n(y, z)$ be the local concentration of the hydrogen ions. Here, Poisson's equation becomes

$\frac{\mathrm{d}^{2} \psi}{\mathrm{d} y^{2}}=-\frac{e}{\varepsilon} n(y, 0)$,

where $z=0$ is set at the midpoint through the capillary. Substitution of Eq. (2) into the above equation results in

$\frac{\mathrm{d}^{2} \bar{\psi}}{\mathrm{d} y^{2}}=-\kappa^{2} \mathrm{e}^{-\bar{\psi}}$

where the Debye screening parameter $\kappa$ is defined by Eq. (4). For the case of constant surface potential $\zeta$, the boundary conditions for $\bar{\psi}$ are

$y=0: \quad \frac{\mathrm{d} \bar{\psi}}{\mathrm{d} y}=0$,

$y=h: \quad \bar{\psi}=\bar{\zeta}$.

The solution to Eq. (19) subject to Eqs. (20a) and (20b) can be obtained as

$\bar{\psi}=\bar{\psi}_{0}+2 \ln \cos [C-(\kappa h-\kappa y) B]$,

where

$B=\mathrm{e}^{-\bar{\psi}_{0} / 2} / \sqrt{2}$,

$C=\tan ^{-1} \sqrt{\mathrm{e}^{\bar{\psi}} \overline{0}_{0} \bar{\zeta}-1}$,

and now $\bar{\psi}_{0}=\bar{\psi}(0)=\bar{\zeta}-2 \ln \cos C$, representing the potential at the median plane between the slit walls.

For the case of constant surface charge density $\sigma$, the boundary condition given by Eq. (20b) is replaced by

$y=h: \quad \frac{\mathrm{d} \bar{\psi}}{\mathrm{d} y}=\frac{e \sigma}{\varepsilon k T}$,

and the solution for $\bar{\psi}$ given by Eq. (21) is still valid, with

$\bar{\zeta}=1+\frac{e \sigma}{\varepsilon \kappa k T} \operatorname{coth}(\kappa h)$.

For the ionic solution in a capillary slit with a given separation distance between its walls, the magnitude of $\zeta$ is a decreasing function of $\kappa h$ for a constant value of $\sigma$ and the magnitude of $\sigma$ is an increasing function of $\kappa h$ for a constant value of $\zeta$.

The numerical values of the dimensionless potential $\bar{\psi}$ calculated from Eq. (21) are plotted in Fig. 7 as functions of the dimensionless coordinate $y / h$ for several values of the dimensionless parameters $\bar{\zeta}$ and $\kappa h$. Similar to the case of a capillary tube shown in Fig. 2, the value of $\bar{\psi}$ is always negative with its magnitude decreasing with a decrease in $y / h$ when the electric double layers adjacent to the walls are relatively thick for a constant magnitude of $\bar{\zeta}$, and it increases with a decrease in $y / h$ from the given $\bar{\zeta}$ at the walls and becomes positive after $y / h$ is smaller than certain values when the double layers are relatively thin. The value of $\bar{\psi}$ at 


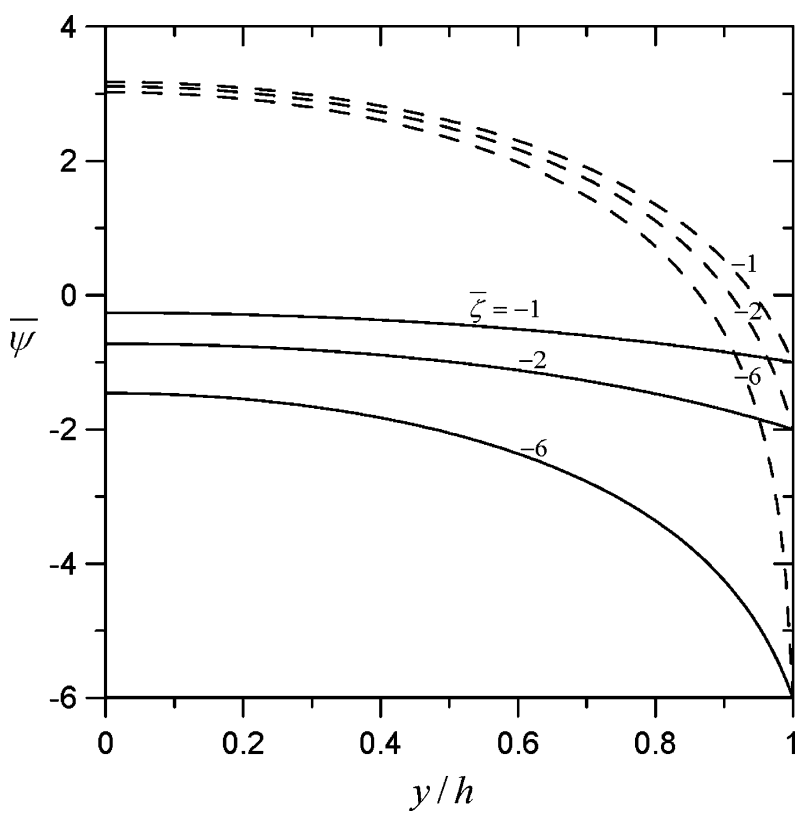

Fig. 7. Plots of the dimensionless potential $\bar{\psi}(y)$ in a capillary slit versus the relative position $y / h$ for various values of $\bar{\zeta}$. The solid and dashed curves denote the cases $\kappa h=1$ and $\kappa h=10$, respectively.

any position decreases with a decrease in the values of $\bar{\zeta}$ and $\kappa h$. Since the adjacent wall area per unit volume of the fluid is smaller in a slit than in a tube, the decay of the electrostatic potential from the capillary walls is faster in a slit than in a tube.

\subsection{Fluid velocity distribution}

The momentum equations for the steady flow of the solution of hydrogen ions in a capillary slit with an applied electric field and ionic concentration gradient parallel to the slit walls are

$\frac{\partial p}{\partial y}+e n \frac{\mathrm{d} \psi}{\mathrm{d} y}=0$

$\eta \frac{\mathrm{d}^{2} u}{\mathrm{~d} y^{2}}=\frac{\partial p}{\partial z}-e n E$

where $u(y)$ is the fluid velocity profile in the tangential direction of decreasing ionic concentration, $p(y, z)$ is the pressure distribution, and $E$ is the applied electric field. Here, the boundary conditions for $u$ are

$y=0: \quad \frac{\mathrm{d} u}{\mathrm{~d} y}=0$,

$y=h: \quad u=0$.

After the substitution of Eq. (2) into Eq. (25a), the pressure distribution can still be determined as the form of Eq. (14), in which $p_{0}$ is the pressure on the midplane between the slit walls and the electrostatic potential distribution $\bar{\psi}(y)$ is given by Eq. (21). Fig. 8 illustrates the result of the normalized pressure $\left(p-p_{0}\right) / n^{\infty} k T$ obtained from Eqs. (14) and (21) as functions of the relative coordinate $y / h$ for various values of the dimensionless parameters $\bar{\zeta}$ and $\kappa h$. Analogous to the result in the case of a capillary tube in Fig. $3,\left(p-p_{0}\right) / n^{\infty} k T$ is positive and an increasing function of $y / h$ for any specified values of $\bar{\zeta}$ and $\kappa h$. This normalized pressure increases with an increase in the magnitude of $\bar{\zeta}$ for given values of $y / h$ and $\kappa h$, and its increase with $y / h$ becomes gentler when either $\kappa h$ or the magnitude of $\bar{\zeta}$ is smaller. Since the decay of the electrostatic potential from the

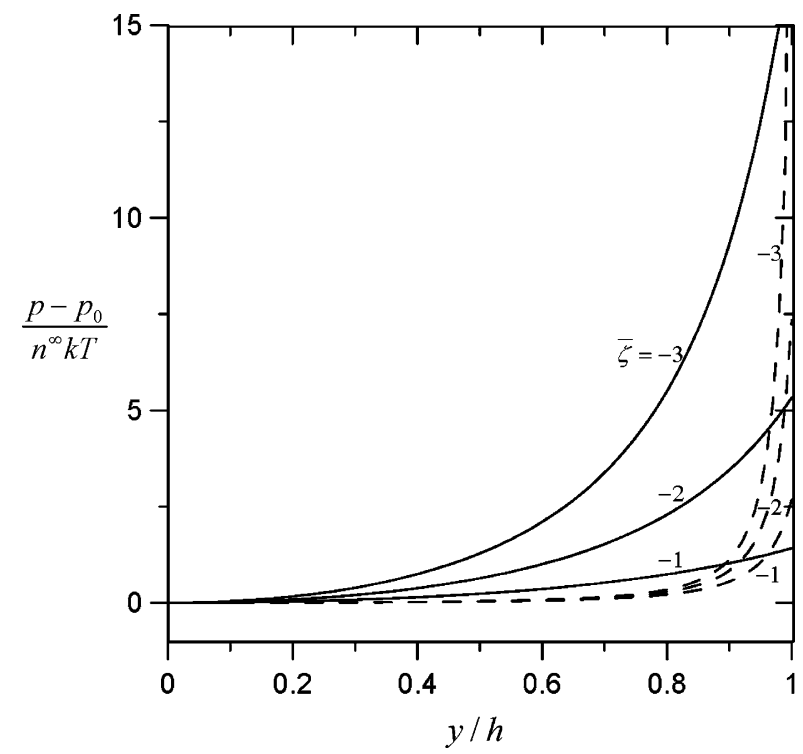

Fig. 8. Plots of the dimensionless pressure $\left(\underline{p}-p_{0}\right) / n^{\infty} k T$ in a capillary slit versus the relative position $y / h$ for various values of $\bar{\zeta}$. The solid and dashed curves denote the cases $\kappa h=1$ and $\kappa h=10$, respectively.

capillary walls is faster in a slit than in a tube, the variation of the pressure with the lateral position is stronger in a slit than in a tube, as Eq. (25a) predicts.

The fluid velocity $u$ can be solved from Eqs. (25b), (26a) and (26b) by the substitution of the ionic concentration distribution of Eq. (2) and the pressure profile of Eq. (14), with a form similar to Eq. (15):

$$
\frac{u}{U^{*}}=(\kappa h)^{2} \int_{1}^{y / h} \int_{0}^{y / h}\left[\left(\mathrm{e}^{-\bar{\psi}_{0}}-\mathrm{e}^{-\bar{\psi}}\right) \bar{N}-\mathrm{e}^{-\bar{\psi}} \bar{E}\right] d \frac{y}{h} d \frac{y}{h},
$$

where $U^{*}$ is defined by Eq. (16). After substituting the electrostatic potential distribution of Eq. (21) and integrating with respect to $y$

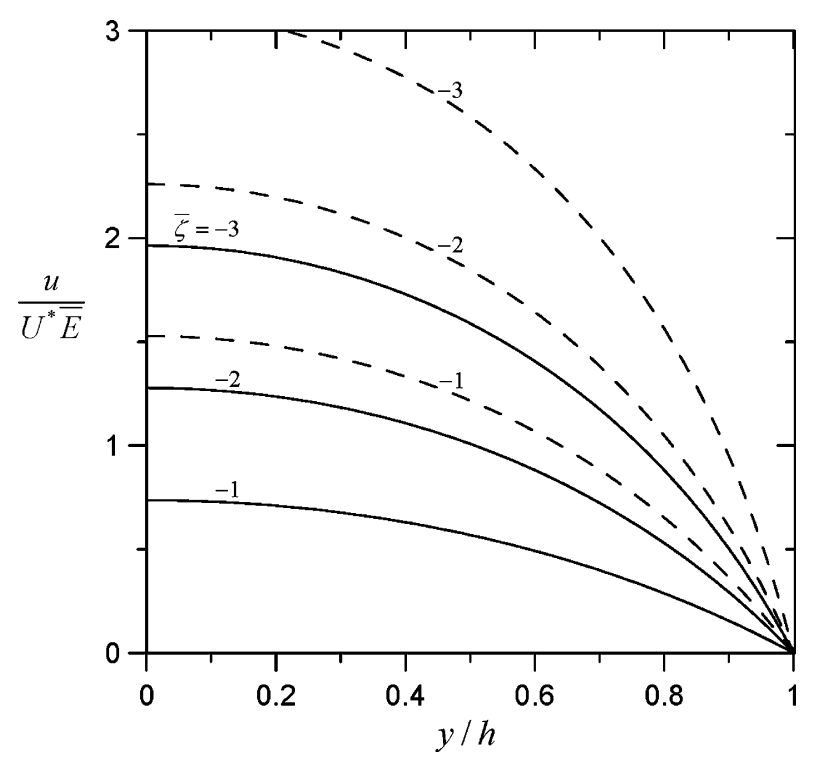

Fig. 9. Plots of the dimensionless electroosmotic mobility $u / U^{*} \bar{E}$ in a capillary slit as calculated from Eq. (28) with $\bar{N}=0$ versus the relative position $y / h$ for various values of $\bar{\zeta}$. The solid and dashed curves denote the cases $\kappa h=1$ and $\kappa h=2$, respectively. 


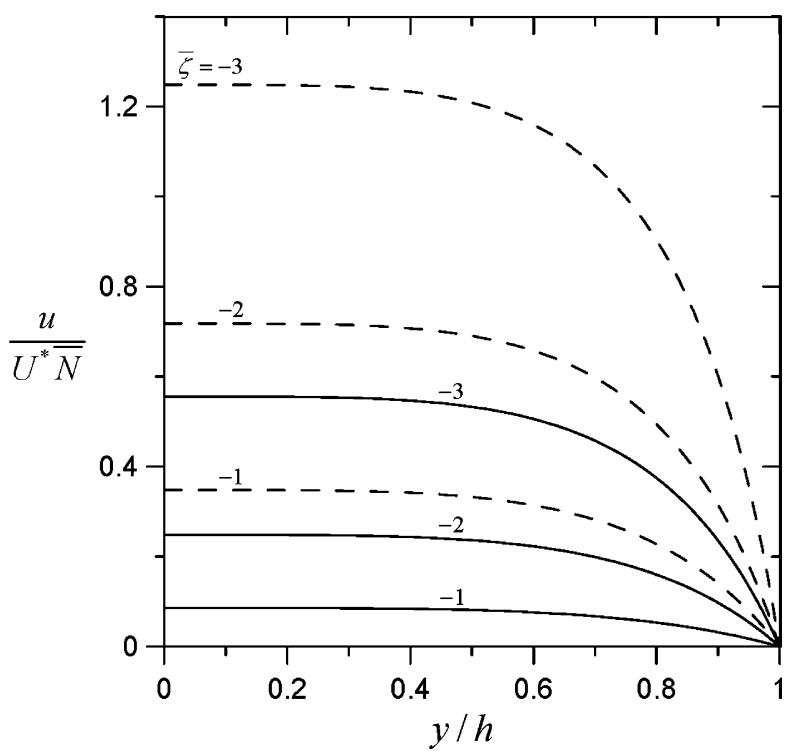

Fig. 10. Plots of the dimensionless diffusioosmotic mobility $u / U^{*} \bar{N}$ in a capillary slit as calculated from Eq. (28) with $\bar{E}=0$ versus the relative position $y / h$ for various values of $\bar{\zeta}$. The solid and dashed curves denote the cases $\kappa h=1$ and $\kappa h=2$, respectively.

twice, the above equation becomes

$$
\begin{aligned}
\frac{u}{U^{*}}= & -\left[(\kappa h)^{2}-(\kappa y)^{2}\right] B^{2} \bar{N} \\
& +2\left\{\ln \frac{\cos [C-(\kappa h-\kappa y) B]}{\cos C}-(\kappa h-\kappa y) B \tan (C-\kappa h B)\right\} \\
& \times(\bar{E}+\bar{N}),
\end{aligned}
$$

where the dimensionless parameters $B$ and $C$ are defined by Eqs. (22a) and (22b).

The average fluid velocity $\langle u\rangle$ over a cross-section of the slit can be expressed as

$$
\langle u\rangle=\int_{0}^{1} u\left(\frac{y}{h}\right) d \frac{y}{h},
$$

which can be obtained by numerical integration after the substitution of Eq. (28).

\subsection{Results of fluid velocity and discussion}

The electroosmotic velocity of the hydrogen ionic solution in the capillary slit is given by Eq. (28) setting $\bar{N}=0$, whereas the diffusioosmotic velocity can be obtained by the same equation taking $\bar{E}=0$. In Figs. 9 and 10, these electrokinetic velocities in dimensionless mobility form are plotted versus the normalized coordinate $y / h$ for several values of the parameters $\bar{\zeta}$ and $\kappa h$. Analogous to the case of a capillary tube discussed in the previous section, both the dimensionless electroosmotic mobility $u / U^{*} \bar{E}$ and diffusioosmotic mobility $u / U^{*} \bar{N}$ decrease monotonically with an increase in $y / h$ and vanish at the no-slip capillary walls. The fluid flows toward the end with lower electric potential or ionic concentration. Both of these electrokinetic velocities are monotonically increasing functions of $\kappa h$ and the magnitude of $\bar{\zeta}$.

The dimensionless average electrokinetic velocity $\langle u\rangle / U^{*} \bar{E}$ of the ionic solution in the capillary slit calculated using Eq. (29) is plotted in Fig. 11 as functions of $\kappa h$ for some values of the parameters $\bar{\zeta}$ and $\bar{N} / \bar{E}$. Again, the contribution from diffusioosmosis to the total fluid flow increases with the ratio $\bar{N} / \bar{E}$ and is quite significant as this ratio is greater than $1 / 2$. In accordance to the electroosmotic and diffusioosmotic velocity profiles illustrated in Figs. 9 and 10, the average fluid velocity increases with an increase in $\kappa h$ and the magnitude of $\bar{\zeta}$ at any value of $\bar{N} / \bar{E}$. Since the viscous retardation exerted on the fluid flow by the capillary walls decreases with a decrease in the adjacent wall area per unit volume of the fluid, the normalized magnitudes of the electroosmotic velocity, diffusioosmotic velocity, and total electrokinetic velocity in a slit is greater than their corresponding magnitudes in a tube.

\section{Concluding remarks}

An analytical study of the effect of diffusioosmosis, in comparison with that of electroosmosis, on the transport of water in the fully hydrated membrane of a PEFC at the steady state is presented in this work. The reactant gases are fed at the same pressure so that no external pressure gradient exists in the membrane and the excess water produced by the chemical reaction at the cathode is drained. A capillary tube or slit with negatively charged walls is chosen to model the interstitial nanochannels in the membrane. It is assumed that the fluid is only slightly nonuniform in the concentration of hydrogen ions along the axial direction of the capillary, but no assumption is made about the thickness of the electric double layers adjacent to the capillary walls. The capillary walls may have either a constant surface potential or a constant surface charge density of an arbitrary quantity. By solving the Poisson-Boltzmann equation and the modified Navier-Stokes equation applicable to the system, the electrostatic potential distribution and the pressure profile under the influence of a constant prescribed concentration gradient of hydrogen ions are determined analytically. The results for the local and averaged electrokinetic velocities of the fluid in the capillary are presented in detail, and the effect of diffusioosmosis on the water transport in the membrane of a PEFC is found to be significant, especially when the macroscopic electric field is low.

Although we discuss the diffusioosmotic effect in the previous sections mainly on PEFCs, with protons as the moving ions, the same analysis can also be applied for other fuel cells with a similar mechanism. For examples, in solid-state alkaline fuel cells, hydroxide ions migrate in anion-exchange membranes, whereas in molten carbonate fuel cells, carbonate ions move in chemically inert

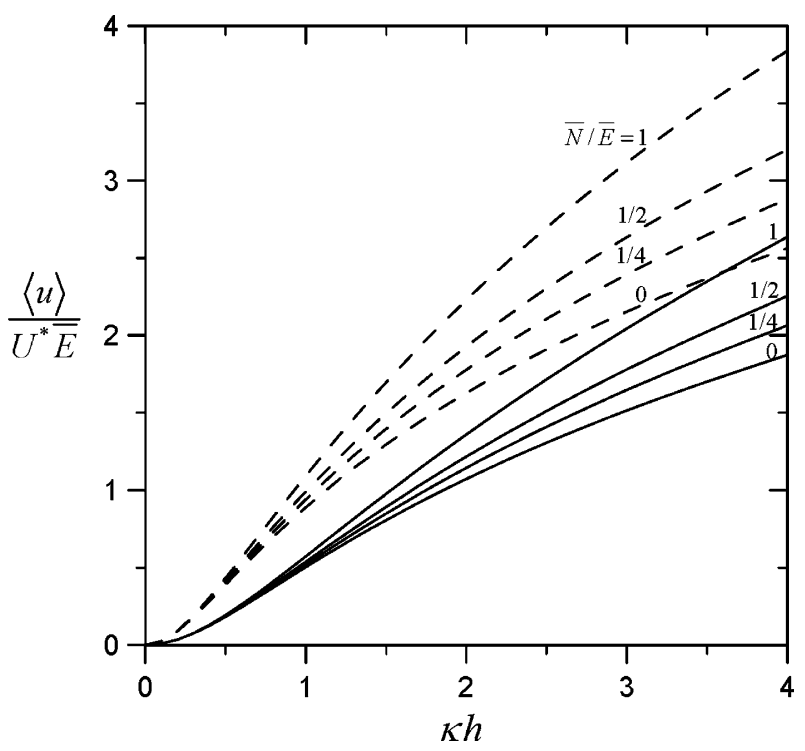

Fig. 11. Plots of the dimensionless average electrokinetic velocity $\langle u\rangle / U^{*} \bar{E}$ in a capillary slit as calculated from Eq. (29) versus the electrokinetic radius $\kappa h$ for various values of $\bar{N} / \bar{E}$. The solid and dashed curves denote the cases $\bar{\zeta}=-1$ and $\bar{\zeta}=-2$, respectively. 
ceramic matrices. The major difference is that the positive hydrogen ions in the membranes are replaced by some negative ions, and the direction of the electrokinetic flow of the fluid will be reversed.

\section{Acknowledgment}

This research was supported by the National Science Council of the Republic of China under Grant NSC95-2221-E-002-281.

\section{References}

[1] S.H. Ge, B.L. Yi, J. Power Sources 124 (2003) 1-11.

[2] F. Barbir, PEM Fuel cells, Elsevier Academic Press, Burlington, MA, 2005.

[3] F.L. Chen, M.H. Chang, C.F. Fang, J. Power Sources 164 (2007) 649-658.

[4] Q.F. Li, R.H. He, J.O. Jensen, N.J. Bjerrum, Chem. Mater. 15 (2003) 4896-4915.

[5] Y. Yin, O. Yamada, K. Tanaka, K. Okamoto, Polym. J. 38 (2006) 197-219.

[6] T.F. Fuller, J. Newman, J. Electrochem. Soc. 140 (1993) 1218-1225.

[7] A.A. Kulikovsky, J. Electrochem. Soc. 150 (2003) A1432-A1439.

[8] M. Eikerling, A.A. Kornyshev, A.R. Kucernak, Phys. Today 59 (2006) 38-44.

[9] T.F. Fuller, J. Newman, J. Electrochem. Soc. 139 (1992) 1332-1337.
[10] T.A. Zawodzinski, J. Davey, J. Valerio, S. Gottesdeld, Electrochim. Acta 40 (1995) 297-302.

[11] D. Weng, J.S. Wainright, U. Landau, R.F. Savinell, J. Electrochem. Soc. 143 (1996) 1260-1263.

[12] M. Ise, K.D. Kreuer, J. Maier, Solid State Ionics 125 (1999) 213-223.

[13] G. Karimi, X. Li, J. Power Sources 140 (2005) 1-11.

[14] S.H. Ge, B.L. Yi, P.W. Ming, J. Electrochem. Soc. 153 (2006) A1443-A1450.

[15] S.S. Dukhin, B.V. Derjaguin, in: E. Matijevic (Ed.), Surface and Colloid Science, Wiley, New York, 1974

[16] D.C. Prieve, J.L. Anderson, J.P. Ebel, J. Fluid Mech. 148 (1984) 247-269.

[17] J.L. Anderson, Annu. Rev. Fluid Mech. 21 (1989) 61-99.

[18] H.J. Keh, S.B. Chen, Langmuir 9 (1993) 1142-1149.

[19] H.J. Keh, Y.K. Wei, J. Colloid Interface Sci. 252 (2002) 354-364.

[20] Y.K. Wei, H.J. Keh, Colloids Surf. A 222 (2003) 301-310.

[21] H.J. Keh, L.Y. Hsu, Microfluidics Nanofluidics, in press.

[22] H.J. Keh, H.C. Ma, Langmuir 21 (2005) 5461-5467.

[23] H.C. Ma, H.J. Keh, J. Colloid Interface Sci. 298 (2006) 476-486.

[24] H.J. Keh, J.H. Wu, Langmuir 17 (2001) 4216-4222.

[25] H.J. Keh, H.C. Ma, Colloids Surf. A 233 (2004) 87-95.

[26] H.J. Keh, H.C. Ma, Langmuir 23 (2007) 2879-2886.

[27] S. Qian, B. Das, X. Luo, J. Colloid Interface Sci. 315 (2007) 721-730.

[28] S.S. Dukhin, Adv. Colloid Interface Sci. 44 (1993) 1.

[29] H. Ohshima, J. Colloid Interface Sci. 247 (2002) 18-23. 